\title{
L'anglais de spécialité est un anglais de la liberté
}

The tail is wagging the dog

Jacques Coulardeau

\section{(2) OpenEdition}

Journals

Édition électronique

URL : http://journals.openedition.org/asp/4312

DOI : $10.4000 /$ asp.4312

ISSN : 2108-6354

Éditeur

Groupe d'étude et de recherche en anglais de spécialité

Édition imprimée

Date de publication : 1 octobre 1993

Pagination : 333-344

ISSN : 1246-8185

Référence électronique

Jacques Coulardeau, «L'anglais de spécialité est un anglais de la liberté », ASp [En ligne], 2 | 1993, mis en ligne le 05 mars 2014, consulté le 02 mai 2019. URL : http://journals.openedition.org/asp/4312 ; DOI : 10.4000/asp.4312

Ce document a été généré automatiquement le 2 mai 2019.

Tous droits réservés 


\title{
L'anglais de spécialité est un anglais de la liberté
}

The tail is wagging the dog

\author{
Jacques Coulardeau
}

1 J'ai envie dans ce papier de prendre à contre-pied une vision étroite que beaucoup d'enseignants ont de l'anglais de spécialité. Il est courant de voir certains de nos collègues, particulièrement ceux qui arrivent dans les lycées techniques sans aucune préparation à ce nouvel anglais qui va les assaillir, se réfugier dans un anglais général qui est en fait au mieux touristique et au pire littéraire, ou bien s'enfuir vers le classique et moderne dès que la porte s'ouvre. J'ai envie ici de dire et d'expliquer pourquoi cet anglais de spécialité est un anglais de liberté, un espace de liberté dans notre système.

\section{Les contraintes}

2 Je ne nie pas que l'anglais de spécialité soit une langue et donc un ensemble de règles contraignantes. Je ne nie pas que l'anglais de spécialité soit un ensemble de règles plus contraignantes encore que l'anglais littéraire car la visée de communication univoque impose d'éviter toute ambiguïté et donc tout jeu sur les mots, sur les structures, bref de ne pas faire avec la langue ce que l'on veut. Si vous avez de la liberté une vision dironsnous anarchiste, ou pire encore individualiste, l'anglais de spécialité vous fera souffrir.

3 C'est là la caractéristique première de toute langue dont le but est la communication technique ou scientifique : ne pas jouer avec les mots car on ne joue pas avec les choses référentielles auxquelles ils renvoient. Autant dans un texte général je peux appeler « $a$ car» " an automobile », " a motor car», " a passenger car », " an auto » ou quelques autres mots encore, autant dans un texte technique «a computer » est «a computer» et rien d'autre. L'anglais technique «calls a spade a spade» et n'a pas le temps ni le désir de l'appeler « a digging apparatus » ou « a hole-making device ».

4 De plus, cet anglais vise à une compréhension sécure de la langue, c'est-à-dire à une compréhension qui assure au technicien, à l'ouvrier ou à l'ingénieur qu'il ne comprendra 
pas de travers l'instruction ou qu'il ne donnera pas une instruction tordue, de telle sorte qu'aucun incident ni aucun accident ne se produiront sur la machine ou dans son environnement du fait de cette mécompréhension, de cette mauvaise communication. Airspeak est l'archétype de ce langage.

Cela est d'autant plus important que l'on vise à ce niveau non plus la communication mondaine dans un salon ou dans une salle de classe, mais la communication mondiale de gens qui par ailleurs ne sont même pas capables de tenir une conversation simple sur le temps qu'il fait ou la guerre du Golfe en anglais.

6 Si on regarde l'anglais de spécialité de ce seul point de vue, on a un carcan si lourd que c'est un lieu d'enfermement et d'écrasement, de frustration et d'aliénation. Cela se retrouve souvent chez nos jeunes collègues à qui on montre un texte sur une configuration quelconque de machine et qui pose cette question ô combien naïve :

Mais qu'est-ce qu'on peut dire dessus?

C'est contre cette position que je veux m'exprimer.

\section{Liberté professionnelle pour les étudiants}

Nos étudiants auront des besoins d'anglais que nous pouvons très clairement définir. Que ce soient nos étudiants scientifiques ou nos étudiants techniques, ils auront besoin d'une bonne connaissance de l'anglais de spécialité de leur champ d'activité, avec l'anglais de l'informatique, de la communication et de plus en plus du multimédia comme terre commune à tous. Ils auront des besoins encore plus spécifiques. Ils devront savoir lire des notices techniques, des présentations de matériels, des dossiers de présentationdiscussion d'une technique ou d'un problème technique se posant à un moment donné. Ils devront être capables d'en faire une synthèse écrite ou orale dans divers lieux professionnels et sur divers médias, particulièrement le média informatique. Ils devront dominer l'écriture des rapports sur des productions ou des fonctionnements d'équipements. Ils devront savoir prendre une conversation téléphonique dans leur champ d'activité pour de la maintenance à distance, pour de l'échange d'information, pour d'innombrables objectifs nécessitant une communication rapide et directe, c'est à dire conversationnelle. L'ordinateur qui gouverne les éclairages du Théâtre National de la Région Nord-Pas-de-Calais est suédois. La maintenance courante se fait par téléphone, et bien sûr l'anglais y est capital. C'est cet oral qui sera nécessaire en premier. Les techniciens de Bull à Villeneuve d'Ascq sont constamment au téléphone avec les branches étrangères, américaines ou autres, et la conversation se fait en anglais. Ce type d'oral est difficile, car il est entouré d'un fort bruit ambiant (le bruit du bureau), il n'est pas assuré d'un son que nous dirons digital (parasites, friture, interférences, statics, etc.), il est désincarné car sans aucun élément de gestique ou d'expressivité faciale ou corporelle. Voilà quelques-uns des besoins prioritaires auxquels nous devons préparer nos étudiants scientifiques et techniques, qui sont aujourd'hui, et de loin, les plus importants en nombre et surtout en taux de croissance. Certes l'anglais, dit général, est utile, mais sa syntaxe n'est pas bien différente de celle de l'anglais technique, même si elle est plus libre, et le reste n'est que du lexique, et là, à vrai dire, le lexique est fort réduit. Pour dire que l'on apprend aussi bien l'anglais dans l'anglais de spécialité que dans l'anglais dit général. 
9 Le professeur d'anglais dans ce domaine doit préparer ses étudiants, que ce soit en Lycée professionnel, en Lycée technique, en BTS, en IUT, en Écoles d'ingénieurs, ou simplement dans les Universités (médecine, physique, mathématique, chimie, biologie, etc.) à être capable d'affronter avec succès ces situations de communication et ces discours bien clairement définis. Le professeur d'anglais de spécialité assure la liberté de ces futurs techniciens, ingénieurs et scientifiques. L'anglais de spécialité est un des outils de leur liberté.

10 Autrefois, on avait tendance à définir la culture comme étant ce qui reste quand on a tout oublié. C'est vrai d'une culture éthique, voire philosophique dont le pouvoir premier est le mode de pensée. Cela est toujours vrai, même dans les domaines scientifiques les plus spécialisés. Mais cela est parfaitement faux dans les domaines scientifiques et techniques si on l'applique à la lettre. Feriez-vous confiance à votre médecin s'il vous disait que sa culture médicale c'est ce qui reste quand il a tout oublié de son savoir médical? Prendriez-vous ses médicaments? Obéirez-vous à ses instructions? Il faut redéfinir la culture aujourd'hui. La culture est ce qui se construit dans l'esprit de l'homme au fur et à mesure qu'il apprend à résoudre en autonomie des problèmes techniques et scientifiques de plus en plus complexes et mettant en cause de plus en plus de savoir. Certes, la culture n'est pas ce savoir, mais elle n'est rien sans ce savoir.

11 Il me plait parfois de me demander ce que c'est la culture d'une informaticienne. Souvent, on se gausse de ces hommes et de ces femmes qui savent sur le bout du doigt hypernaviguer dans n'importe quel programme de n'importe quelle machine, qui ne lisent que des revues spécialisées, et qui ne lisent rien d'autre, qui ne vont pas au théâtre, qui ignorent l'art lyrique qui ne savent pas ce que c'est un cinéma et ne connaissent comme tout écran que celui de leur ordinateur, ignorant même celui de la télévision. Il est vrai que certains faits dans notre société poussent à une telle déshumination du technicien. Mais quel pouvoir énorme il possède au bout de ses doigts entièrement gouvernés par son cerveau! Nous sommes des handicapés, des petits enfants face à ce pouvoir qui nous échappe. Et ce pouvoir nécessite en profondeur une drôle de puissance intellectuelle. Car il s'agit bien d'un pouvoir qui permet de résoudre des problèmes posés de façon juste et sans erreur. Je considère que le scientifique ou le technicien d'aujourd'hui est le nouveau philosophe de notre époque moderne. Il n'est pas celui qui pose des enjeux verbaux ou référentiels, éthiques ou méthodologiques qui se posent à l'homme dans la vie de tous les jours, dans la rue ou dans son salon, en dehors de tout appareillage. Il pose le problème majeur, non pas en termes verbaux, mais en termes d'action, de la relation entre l'homme et la machine, entre les hommes par l'intermédiaire de la machine. Non seulement il pose ces problèmes, mais encore il les résout d'une façon ou d'une autre, car chaque ligne d'un programme dans une telle machine est une solution apportée à un problème posé à ce niveau.

Quand on sait que l'anglais de spécialité est central pour ce technicien, ce scientifique, cet ingénieur, on comprend que la possession de cet anglais est aussi un ferment particulièrement important de liberté pour ces hommes et de liberté pour l'humanité.

13 J'ai dit liberté et non libération. Nous sommes en train de dépasser la vision de la libération de l'humanité et de l'homme que nous nous étions construite au dix-neuvième siècle. Dans nos sociétés, et je prends la précaution de laisser un peu en suspens les sociétés dites sous-développées, il ne s'agit plus tant de permettre à des hommes d'acquérir des libertés qu'ils n'ont pas par un processus social de libération. Il s'agit d'inciter les hommes, tous les hommes, à acquérir par leur propre effort des libertés 
nouvelles que sont le savoir technique et scientifique, le savoir qui seul importe pour la profession et donc la position de l'homme dans la société. La société doit veiller à ce que tous les hommes, à capacités égales, aient les mêmes chances d'accès à ces outils de liberté. La société doit veiller à ce que les hommes qui ont des capacités inférieures puissent sur une ou deux générations rattraper tout ou partie de leur retard. Mais le processus d'acquisition de ces libertés qu'est le savoir technique et scientifique est un processus ou l'individu est face à lui-même plus encore que face aux autres ou aux institutions. Je ne saurais acquérir qu'un savoir que je vise personnellement. C'est là la clé de ma motivation. C'est là la clé de mon processus d'acquisition de la liberté, de construction de ma liberté. Il est impensable aujourd'hui d'avoir dans les institutions d'acquisition du savoir des syndicats d'étudiants qui revendiqueraient le droit pour tous les étudiants d'avoir leurs examens et leurs diplômes, comme autrefois les syndicats ouvriers revendiquaient le droit pour tous les ouvriers d'avoir des congés payés ou un salaire décent.

On nous dit souvent que c'est là un individualisme regrettable. Nous reconnaissons l'individualisme, mais nous ne le regrettons pas car l'acquisition du savoir n'est qu'un processus personnel et individuel. Je suis seul face au savoir que je vise, même si ma motivation et donc ma visée d'un certain savoir dépendent énormément de mon entourage, du soutien que j'y trouve, de l'encouragement que j'y sens, et de l'aide que j'y reçois.

15 Aujourd'hui l'anglais de spécialité est une clé dans ce processus. C'est une des clés de la liberté professionnelle, sociale, individuelle et culturelle des hommes et des femmes. Nous sommes loin de la simple capacité de pouvoir aller à Londres et de trouver son chemin de Victoria Station à Buckingham Palace.

\section{Liberté professionnelle des enseignants}

La première des libertés professionnelles de l'enseignant, c'est l'absence de référentiel ou de textes réglementaires.

Pour le BTS Audiovisuel, voilà le référentiel :

Éléments morphologiques et syntaxiques inventoriés au titre du programme grammatical de base (B.O. spécial du 29 avril 1982, p. 29)

Vocabulaire essentiel (en tenant compte des listes de fréquences); vocabulaire technique propre au domaine de l'audiovisuel

Perfectionnement phonétique

L'épreuve change cette année mais le référentiel non. On voit que pour le professeur concerné, sa responsabilité et sa liberté sont entièrement engagées. Il peut faire tout ce qu'il veut car ce qu'il doit faire est aussi peu précis qu'un vide complet. Le texte de 1982 est antérieur à l'existence même de ce BTS et relève du programme de révisions dans le second cycle secondaire.

La deuxième des libertés du professeur de langue, c'est l'absence de manuels sérieux dans nos domaines techniques. Certes, nous pouvons trouver ici ou là un lexique, un manuel dit général dans le domaine technique, une grammaire de révision, parfois un manuel plus pointu comme celui d'Arlette Dechet (1988). Mais en général, nous sommes nus et désemparés devant le travail à faire car les aides pédagogiques sont inexistantes. 
D'abord les lexiques spécialisés : il en existe, sous forme de livres de vocabulaire ou de dictionnaires bilingues. Ils sont nombreux dans le domaine tertiaire. Ils sont très peu nombreux dans le domaine technique industriel. Mais il pose un mythe comme premier axiome méthodologique et «culturel»: la langue technique c'est du lexique. C'est une erreur, nous ne le dirons jamais assez.

Ensuite les manuels inter-référence. Par exemple le LAST d'Ellipses, ou quelques autres. Là on trouve des textes. On trouve même chez certains des chapitres de remise en ordre syntaxique. Notons que ces textes sont toujours anciens dans des domaines qui évoluent si vite qu'on ne peut pas faire d'une année sur l'autre les mêmes documents, sauf à prétendre que la langue ne bouge pas. Au niveau technique, quand on sort d'une école, le matériel sur lequel on a appris est dépassé. La durée du cycle de rotation dans le domaine de la technique de pointe est de l'ordre de deux ans, la durée d'un BTS ou d'un IUT. C'est la même chose dans la langue. En l'espace de deux ans, tout le corpus des abréviations et du lexique de pointe s'est transformé. Le professeur se doit de lire des revues avancées dans le domaine et de choisir année après année des documents qui vont bien au cœur de la langue de spécialité telle qu'elle se pratique au moment où il l'enseigne, ou au plus six ou neuf mois avant. Un manuel est tout au plus un outil de travail pour l'étudiant qui y trouvera assez rapidement le lexique qu'on dira élémentaire permanent, si on peut poser un tel concept.

2 Le professeur est libre devant le champ technique qu'il aborde.

Au niveau de la syntaxe, il n'existe rien de sérieux. LAST d'Ellipses (1983) est totalement inadéquat. Il ne permet aucune vue synthétique et claire sur le moindre point de syntaxe de l'anglais de spécialité. Mais j'aimerais insister sur deux manuels.

As ou Like? de Philippe Laruelle (1993). Le sous-titre me paraît bien loin de la vérité du livre : « 50 problèmes résolus de grammaire anglaise ».

Si on regarde le détail, on trouve souvent de bonnes choses, mais la volonté de couper les problèmes les uns des autres donne un ensemble éparpillé difficile à utiliser par l'étudiant, et par le professeur. Il est vrai que l'auteur fait un effort de compréhension linguistique des choses, mais il n'y a jamais une vue synthétique des grands points de la grammaire.

26 Prenons par exemple les modaux. Ils sont explosés en onze points successifs, tous, car c'est la règle du manuel, présentés en oppositions binaires. Il se laisse aller à une comparaison systématique avec l'allemand, ce qui est peu intéressant. Prenons le premier chapitre : «CAN ou MAY?»

27 Il part d'emblée d'un tableau que je n'oserais jamais donner à un étudiant car ce tableau (voir tableau 1) va fixer visuellement dans la mémoire de l'étudiant une erreur majeure.

Tableau 1

\begin{tabular}{|l|l|l|l|}
\hline & Français & Anglais & Allemand \\
\hline Capacité & il peut le faire & He can do it & Er kann es tun \\
\hline Permission & Tu peux quitter la table & $\begin{array}{l}\text { You may leave the } \\
\text { table }\end{array}$ & Du darfst den Tisch verlassen \\
\hline
\end{tabular}




\begin{tabular}{|l|l|l|l|}
\hline Éventualité & $\begin{array}{l}\text { Je peux me tromper, mais } \\
\text { ça m'étonnerait }\end{array}$ & $\begin{array}{l}\text { I may be wrong, but } \\
\text { I'd be surpised }\end{array}$ & $\begin{array}{l}\text { Ich mag mich irren, es würde } \\
\text { aber mich wundern }\end{array}$ \\
\hline
\end{tabular}

Laruelle 1993 : 72

Non, il faut le dire avec force, MAY n'est pas le modal de la permission. Avec cette valeur il est aujourd'hui pratiquement cantonné à la forme interrogative et encore il fait un peu archaïque. Il est vrai que l'on trouve MAY avec cette valeur dans les notices techniques à la forme affirmative. Mais il est infiniment plus juste de poser dans la mémoire visuelle de l'étudiant MAY comme le modal de l'éventualité avec une note ultérieurement qu'il peut dans certaines notices techniques reprendre une valeur ancienne de permission. Il est sûr que l'entier du chapitre de Laruelle est ensuite une discussion de ce tableau et de cette valeur de MAY. Mais mon reproche c'est que la mémoire visuelle de l'étudiant est infiniment trop puissante pour que je pose d'emblée à ce niveau là une telle "erreur ", même si ensuite je la discute. C'est le visuel qui restera dans la mémoire opératoire de l'étudiant qui aura alors tendance à considérer CAN avec la valeur de permission comme une évolution plus ou moins fautive.

Je critique dans ce manuel, non pas les fautes qui n'y sont en définitive pas, mais la présentation par regroupement binaire des formes qui va systématiquement induire en erreur l'étudiant et donc lui faire se poser dans sa propre tête des erreurs, surtout avec les aides visuels (tableaux) qui renforcent terriblement ces inductions erronées.

Le second c'est Grammaire anglaise pour techniciens de Jean-Paul Bernard (1992).

Autant Laruelle ne se trompait pas quand on lisait tout, mais présentait les choses comme pouvant induire des erreurs, autant J.-P. Bernard pose les erreurs comme des vérités.

MAY exprime la permission, la possibilité qui est offerte de faire quelque chose.

32 On ne pose même pas la valeur de probabilité de MAY. Et on dit :

Dans certains contextes CAN peut avoir un sens très voisin de MAY

CAN exprime la capacité de faire quelque chose.

On ne pose jamais les trois valeurs de cette capacité: physique, intellectuelle et permissive. Cela tient au fait que l'on pose les sujets de ces modaux comme étant des objets, sans voir que l'anglais de spécialité ne réduit pas ces modaux à ces emplois, et sans voir les conséquences que ces emplois ont sur la valeur. Plus loin on pose :

MAY exprime la probabilité relative... MIGHT exprime une notion voisine de celle dont rend compte MAY mais comporte une nuance de probabilité moindre.

4 On met ainsi la valeur épistémique de base de MAY en position seconde, avec tout ce que cela induit d'erreur. Et on se trompe complètement sur MIGHT qui peut tout aussi bien exprimer un plus ou un moins de probabilité.

Cela tient au fait que l'on n'oppose pas radical et épistémique. Ce trait est commun aux deux manuels. Le premier, dans sa dimension comparativiste avec l'allemand, est piégé par le fait que l'allemand oppose des éléments lexicaux différents pour le radical et pour l'épistémique. Laruelle lexicalise le problème. Bernard ignore purement et simplement le problème. Les deux n'expliquent pas la logique de la langue anglaise et donc sa dynamique et ne donne pas l'outil intellectuel nécessaire pour que l'étudiant reconstruise son savoir et ainsi ait un vrai pouvoir sur ce savoir. 

le savoir technique. Ils pensent aussi beaucoup plus visuellement que les autres. Je suis toujours étonné par l'extraordinaire dimension visuelle de la pensée scientifique et technique. Banal que de dire cela quand on parle de géométrie. Mais c'est tout aussi vrai en physique ou en chimie, en algèbre ou en trigonométrie, et bien sûr en informatique ou en technique vidéo. Je pense que la pensée visuelle doit être prioritairement développée chez les jeunes dans les années qui viennent. Si l'école ne le fait pas, la vie le fera, que ce soit la télé, l'ordinateur, les innombrables écrans qui vont s'installer partout dans notre vie. Mais plus encore, l'école le fera sans le savoir. Tous les grands sujets scientifiques et techniques ne fonctionnent que visuellement. Ils forment donc la pensée visuelle sans s'en rendre compte. Je propose que l'on devienne conscient de cela et que l'on en fasse un objectif prioritaire. Nous, les professeurs de langue, avons été prioritairement formés à la pensée linguistique, c'est-à-dire, une pensée qui ne passe que par le mot, ou au plus, le signifiant linguistique qui peut être à la fois visuel (écrit) ou auditif (langue orale). Nous devons apprendre à fonctionner de façon visuelle. On ne doit plus présenter les problèmes comme Bernard, c'est-à-dire avec les gloses, mais comme Laruelle, avec des tableaux comme support des gloses ultérieures. Et c'est là que nous sommes capables d'arriver à une nouvelle liberté : nous libérer de la dictature de la glose pour aller vers le tremplin de la représentation. Toute grande science fonctionne sur des modèles et la réalité virtuelle va démultiplier ces modèles et leur opérativité. Il serait plutôt contradictoire que l'on enseigne l'anglais de la réalité virtuelle mais avec le carcan linguistique d'une pensée de la glose. Je considère que la pensée de la glose est une gangue qui enferme et freine la liberté de l'élève, mais aussi du professeur.

On nous dit souvent qu'il y a des gens qui sont visuels et d'autres non. C'est juste, mais c'est regrettable pour ceux qui ne le sont pas. Ils sont déphasés dans un monde qui devient de plus en plus visuel. En fait, ce sont des visuels qui s'ignorent, des visuels victimes d'un mode de pensée plus anciens dans ce qui est pour eux leur pensée abstraite. Je considère que la pensée abstraite sera démultipliée par la libération de la pensée visuelle chez l'homme.

39 C'est là que la discussion a révélé un point essentiel. Nous nous devons d'enseigner la langue qui sera utile aux techniciens, ingénieurs et scientifiques que seront nos étudiants. Nous devons donc enseigner l'anglais de spécialité et rien d'autre. Il est donc nécessaire d'avoir à notre disposition une description la plus exacte possible de l'anglais de spécialité ou des anglais de spécialité dont nous avons besoin. Il faut expurger de cette description des choses qui relève d'un autre anglais, en particulier de l'anglais littéraire, mais surtout de normes linguistiques anciennes qu'un certain nombre de descriptions de l'anglais «normé » continuent à propager malgré toutes les erreurs qu'elles peuvent provoquer: la discussion s'est appuyée sur la nécessité d'abandonner la présentation prioritaire de «MAY» comme exprimant la permission, et de «SHOULD» comme exprimant le conditionnel. Ce ne sont là que deux exemples. De la même façon, il faut enseigner le subjonctif dans certains domaines car il est indispensable dans l'anglais scientifique. Ce qui nous étonne énormément, c'est que la question du réalisme de la description de l'anglais que l'on enseigne, réalisme qui veut dire la conformité avec le 
discours visé, soit encore une question, car pour nous c'est une porte ouverte. Il nous faut revoir notre enseignement de la langue en fonction de la langue exacte que nous devons enseigner. Même si Byte parle une langue barbare, c'est cette langue que des informaticiens, industriels ou non, devront posséder, c'est à dire comprendre et pratiquer. Notre jugement de valeur sur tel ou tel anglais de spécialité, aussi peu ou bien fondé qu'il soit, n'est en rien réaliste ou pertinent.

\section{Conclusion}

J'ai entièrement centré mon exposé sur le mot de liberté. Il est temps maintenant d'élargir un peu le discours. On a parlé de pensée visuelle. Il me semble que l'approche linguistique la plus visuelle aujourd'hui, c'est la psychomécanique qui cherche à produire des modèles visuels de tous les phénomènes qu'elle étudie. C'est l'école linguistique qui prêche depuis le début la libération de la glose.

41 J'ai parlé de liberté du technicien. Il faudrait parler de liberté de la langue technique. Ce serait un nouvel exposé : une langue technique, dans ses formes les plus avancées est marquée par une création lexicale exubérante et par une licence syntaxique parfois des plus inspirée. C'est la langue de la métaphore et de la métonymie. Aujourd'hui on ne parle plus que de "desktop", "laptop», "palmtop", "notebook», etc. Le premier est une métonymie de "desktop computer ». Les autres ont, pour certains du moins, été créés directement, sans même passer par la forme complète. La métonymie est devenue créatrice directement au niveau morphologique. On pourrait montrer des fonctionnements identiques au niveau syntaxique.

Enfin, nous sommes ici didacticiens. Tout mon discours se fonde sur une volonté d'être efficace dans notre enseignement, une efficacité nouvelle, définie comme devant produire des connaissances linguistiques assurant la sécurité du technicien dans son travail, dans sa vie professionnelle, dans la vie tout court qu'il aura et dont l'activité professionnelle sera la part essentielle. Il est bien sûr que je me place dans une approche cognitive, mais non pas une approche cognitive sémantique comme Langacker ou Fillmore, mais une approche cognitive syntaxique comme Guillaume et Tesnière. Ici cette approche pose un enjeu scientifique fondamental. Il s'agit de redéfinir la limite entre syntaxe et sémantique, posant bien que cette limite n'est en rien naturelle mais relève absolument de l'axiomatique méthodologique du scientifique. Nous sommes confrontés aujourd'hui à un nouveau choix saussurien, j'entends, redéfinir l'axiome méthodologique sans lequel nous ne serons pas capables de comprendre le fonctionnement linguistique : où devonsnous poser la frontière entre la syntaxe et la sémantique?

Mais là nous sommes bien loin de notre sujet, et pourtant si proche. C'est la liberté du scientifique que de pouvoir déplacer les axiomes sur lesquels il travaille. Je dis que notre approche de l'anglais de spécialité nous pousse terriblement à faire cette nouvelle révolution saussurienne dans les plus brefs délais. Quelle liberté que celle-là qui s'appuie sur des faits aussi simples que la valeur de MAY dans les notices techniques. 


\section{BIBLIOGRAPHIE}

Bernard, Jean-Paul. 1992. Grammaire anglaise pour techniciens. Paris : Dunod.

Bosworth-Gérôme, Sally, Colette Helye-Lebas et Robert Marret. 1983. LAST, Lire l'anglais

scientifique et technique. Paris : Ellipses.

Dechet, Arlette. 1988. Goto, l'anglais informatique pour communiquer. Paris : Ellipses.

Laruelle, Philippe. 1993. As ou Like? Paris : Ellipses.

\section{RÉSUMÉS}

Malgré toutes les contraintes que l'anglais de spécialité représente, contraintes syntaxiques, lexicales, grammaticales, discursives ou même pragmatiques, contraintes qu'il ne s'agit pas ici d'examiner, nous voulons montrer combien l'anglais de spécialité est un lieu de liberté. D'abord un lieu de liberté pour l'apprenant, futur technicien, ingénieur ou scientifique, qui acquiert en même temps que cet anglais, un outil qui lui sera indispensable et incontournable dans son acquisition de savoir et dans son futur métier. C'est un outil de sa liberté professionnelle, et une garantie, par la connaissance "sécure" qu'il se doit d'avoir de cet anglais, qu'il ne sera pas responsable d'un accident ou d'un incident plus ou moins grave. Pour l'enseignant, l'anglais de spécialité est aussi un lieu de liberté, d'abord parce que les référentiels sont insuffisants, ensuite parce les manuels sont inexistants ou fort réduits dans le domaine industriel qui nous intéresse ici, enfin parce qu'une pédagogie de l'anglais de spécialité n'existe pas et reste à faire sur la base d'une description exacte de cet anglais et non de la description d'un anglais britannique plus ou moins littéraire plutôt ancien. C'est là la responsabilité et la liberté pédagogique et didactique du professeur : il doit tout faire lui-même.

In spite of all the syntactical, lexical, grammatical, discursive or even pragmatic limitations ESP represents and which will not be examined here, we would like to show how much ESP is a field of freedom. First, a field of freedom for the students, future technicians, engineers or scientists, who receive, at the same time as they receive that English, an unavoidable and indispensable tool both in their learning and in their future jobs. It is a tool for their professional freedom, and a guarantee, in the secure knowledge they must have of it, that they will not cause any light or serious incident or accident. For the teacher, ESP is also a field of freedom. First because classsyllabi are worthless. Then, because text-books are non-existent or very light in the field of industrial ESP we are here concerned with. Finally because an ESP pedagogy does not exist and remains to be devised on the basis of an exact description of this ESP and not of some British, more or less literary, rather old type of English. It is the teacher's responsibility and both pedagogical and didactic freedom: he must do everything all by himself.

\section{INDEX}

Mots-clés : didactique, liberté, pédagogie, responsabilité

Keywords : didactics, freedom, pedagogy, responsibility 
AUTEUR

JACQUES COULARDEAU

Lycée Jean Rostand, Roubaix et UFR LEA, Université Lille 3. dondaine@orange.fr 